\title{
SELF-MAPS OF CLASSIFYING SPACES OF COMPACT SIMPLE LIE GROUPS
}

\author{
STEFAN JACKOWSKI, JAMES E. MCCLURE, AND BOB OLIVER
}

\begin{abstract}
We describe here the set $[B G, B G]$ of homotopy classes of self-maps of the classifying space $B G$, for any compact connected simple Lie group $G$. In particular, we show that two maps $f, f^{\prime}: B G \rightarrow B G$ are homotopic if and only if they are homotopic after restricting to the maximal torus of $G$; or equivalently if and only if they induce the same homomorphism in rational cohomology. In addition, we identify the homotopy types, up to profinite completion, of the components of the mapping space $\operatorname{map}(B G, B G)$.
\end{abstract}

The most central concern of homotopy theory is the classification, up to homotopy, of maps between topological spaces. It has long been suspected that maps between classifying spaces provide a particularly favorable special case of this problem, in which explicit results can be expected. In this paper we announce a complete classification of the self-maps of the classifying space $B G$, when $G$ is any compact connected simple Lie group.

When $G$ and $\Gamma$ are arbitrary compact Lie groups, then $[B G, B \Gamma]$ will denote the set of unbased homotopy classes of maps from $B G$ to $B \Gamma$. It is natural to ask how closely this set is related to the set $\operatorname{Hom}(G, \Gamma)$ of homomorphisms from $G$ to $\Gamma$. For any inner automorphism $\alpha \in \operatorname{Inn}(\Gamma), B \alpha$ is homotopic to the identity on $B \Gamma$. It is thus convenient to write $\operatorname{Rep}(G, \Gamma)=\operatorname{Hom}(G, \Gamma) / \operatorname{Inn}(\Gamma) ;$ and ask when the map

$$
B: \operatorname{Rep}(G, \Gamma) \rightarrow[B G, B \Gamma] . \quad B(\rho)=B \rho
$$

is a bijection.

When $G$ and $\Gamma$ are both finite (or even discrete), then $B$ is easily seen to be bijective. A much deeper result, due to Dwyer and Zabrodsky [2], says that $B$ is bijective whenever $G$ is a $p$-group, (and $\Gamma$ is compact Lie). This was extended by Notbohm [8] to the case where $G$ is a $p$-toral group: i.e., where $G$ has toral identity

Received by the editors January 31, 1989 and, in revised form August 27, 1989. 1980 Mathematics Subject Classification (1985 Revision). Primary 55S37; Secondary $55 \mathrm{~N} 91,55 \mathrm{R} 35$.

The second author was partly supported by an NSF grant. 
component and $\pi_{0}(G)$ is a $p$-group. These theorems depend on the generalized Sullivan conjecture, proven by Miller, Carlsson, and Lannes using different approaches $[16,17,18]$.

Our goal here is to apply the theorems of Dwyer-Zabrodsky and Notbohm to study the sets $[B G, B G]$ when $G$ is a connected, compact, simple Lie group. The first important contribution to this was given by Sullivan [11]. For $G=S U(n)$ and $k$ prime to $n$ !, he showed that the $k$ th power map on $B T$ ( $T \subseteq G$ the maximal torus) can be extended to an "unstable Adams operation" $\psi^{k}: B S U(n) \rightarrow B S U(n)$. Since the $k$ th power map cannot be extended to a homomorphism, $B: \operatorname{Rep}(G, G) \rightarrow[B G, B G]$ is far from surjective in these cases-but the sets $[B G, B G]$ still have very simple descriptions.

Theorem 1. Let $G$ be a compact connected simple Lie group with maximal torus $T$ and Weyl group $W$. Then, for any pair of maps $f, f^{\prime}: B G \rightarrow B G$, the following are equivalent:

(1) $f$ and $f^{\prime}$ are homotopic;

(2) $f \circ B l \simeq f^{\prime} \circ B l: B T \rightarrow B G(l: T \hookrightarrow G)$;

(3) $H^{*}(f ; \mathbf{Q})=H^{*}\left(f^{\prime} ; \mathbf{Q}\right)$.

Furthermore, the image of the injective restriction map

$$
[B G, B G] \nrightarrow[B T, B G]
$$

consists of all maps $B \rho$ for $\rho$ a composite

$$
T \stackrel{t \mapsto t^{k}}{\longrightarrow} G \stackrel{\alpha}{\rightarrow} G,
$$

where $(k,|W|)=1$ and $\alpha \in \operatorname{End}(G)$.

What is new in this theorem is the equivalence of points (1) and (2): note the analogy with the situation for group representations. The equivalence of (2) and (3) follows easily from [8].

The image in $[B T, B G] \cong \operatorname{Rep}(T, G)$ of the restriction map was already known. As mentioned earlier, the existence of maps $\psi^{k}: B G \rightarrow B G$ (for $k$ prime to $|W|$ ) which extend the $k$ th power map on $B T$ was shown by Sullivan when $G=S U(n)$-and his result was extended to arbitrary simple $G$ by Wilkerson [12]. Conversely, Hubbuck $[3,4]$ showed that for every $f: B G \rightarrow B G$, $H^{*}(f, \mathbf{Q})=H^{*}\left(B \alpha \circ \psi^{k} ; \mathbf{Q}\right)$ for some $\alpha$ and $k$; and Ishiguro [5] showed (using [2]) that either $k=0$ or $(k,|W|)=1$. Hubbuck's result also follows as a consequence of Notbohm's theorem.

Various examples constructed by the authors show that Theorem 1 does not generalize to the case of $[B G, B \Gamma]$ for arbitrary 
(or even connected) $G$ and $\Gamma$. For example, there are homomorphisms $\rho, \rho^{\prime}: S U(3) \rightarrow S O(8)$ such that $B \rho$ and $B \rho^{\prime}$ induce the same homomorphism in rational cohomology, but are not homotopic. Also, the Borel construction can be applied to the tangent bundle of any $G$-action on a contractible oriented $n$-manifold to give a vector bundle over $B G$-and hence a map from $B G$ to $B S O(n)$. Thus, the many examples of exotic $G$-actions on $\mathbf{R}^{n}$ can be used to construct exotic elements in $[B G, B S O(n)]$.

Now let $G \supseteq T$ be as in Theorem 1. A map $f: B G \rightarrow B G$ will be said to have "type $k$ " if the following diagram commutes in rational cohomology (or equivalently [8] up to homotopy):

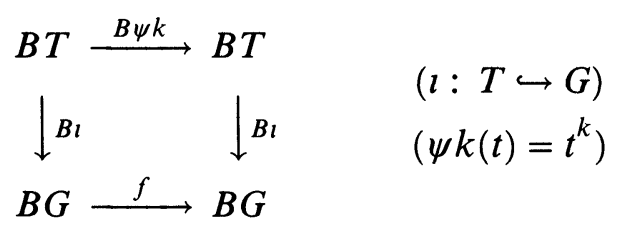

More generally, for any outer automorphism $\alpha \in \operatorname{Out}(G), f$ will be said to have type $(\alpha, k)$ if $B \alpha^{-1} \circ f$ has type $k$.

Note that for simple $G, \operatorname{End}(G) / \operatorname{Inn}(G) \cong\{0\} \amalg \operatorname{Out}(G)-$ since any endomorphism of $G$ is either trivial or an automorphism. Theorem 1 can now be reformulated as follows:

Theorem $1^{\prime}$. For any compact connected simple Lie group $G$ with Weyl group $W$, the correspondence $f \leftrightarrow \operatorname{type}(f)$ defines a bijection

$$
[B G, B G] \stackrel{\cong}{\leftrightarrows}\{0\} \coprod[\operatorname{Out}(G) \times\{k>0:(k,|W|)=1\}] .
$$

Consider the important special case $G=S U(n)$. Note that $\operatorname{Out}(S U(2))=1$, and that $\operatorname{Out}(S U(n))=\{1, \kappa\}$ for $n \geq 3$ (where $\kappa$ denotes complex conjugation). Since $B \kappa$ is an unstable Adams operation of type -1 , Theorem $1^{\prime}$ implies that every self-map of $B S U(n)$ is of type $k$ for some $k$-and that any two maps of type $k$ are homotopic.

The first case of Theorem $1^{\prime}$-when $G=S U(2)$-was proven by Mislin [7]. Later, Dwyer and Mislin [1] gave a complete description of the homotopy type of the space of pointed self-maps of $B S U(2)$ (see also Theorem 2 below).

In the proof of Theorem 1 outlined below, we use not only the theorems of Dwyer, Zabrodsky, and Notbohm about the sets $[B P, B \Gamma]$ for $p$-toral $P$, but also their computation of the $p$ adic homotopy type of the individual connected components of $\operatorname{map}(B P, B \Gamma)$. In order to do this, Dwyer and Zabrodsky introduced the following construction. For any $G$ and $\Gamma$, and any $\rho: G \rightarrow \Gamma$, let $C_{\Gamma}(\rho)$ denote the centralizer of $\operatorname{Im}(\rho)$ in $\Gamma$. Then 
multiplication in $\Gamma$ defines a homomorphism (incl, $\rho): C_{\Gamma}(\rho) \times$ $G \rightarrow \Gamma$, which induces a map

$$
e_{\rho}: B C_{\Gamma}(\rho) \rightarrow \operatorname{map}(B G, B \Gamma)_{B \rho} .
$$

Here, $\operatorname{map}(B G, B \Gamma)_{B \rho}$ denotes the component containing $B \rho$.

As usual, the case where $G$ and $\Gamma$ are both finite is easy: $e_{\rho}$ is then a weak homotopy equivalence for each $\rho$. What is relevant for our results is that when $G$ is $p$-toral, then $e_{\rho}$ induces an isomorphism of $\bmod p$ cohomology for each $\rho$ (see [2 and 8]).

Now assume again that $G$ is connected and simple. When $\rho$ is the trivial or identity endomorphism of $G$, then $e_{\rho}$ takes the form

$e_{0}: B G \rightarrow \operatorname{map}(B G, B G)_{0}$ and $e_{1}: B Z(G) \rightarrow \operatorname{map}(B G, B G)_{1}$, where $Z(G)$ is the center of $G$. For every $k, \alpha, \operatorname{map}(B G, B G)_{\alpha, k}$ will denote the space of maps of type $(\alpha, k)$. For arbitrary $\alpha \in$ $\operatorname{Out}(G)$ and $(k,|W|)=1$, we let $e_{\alpha, k}$ denote the following composite:

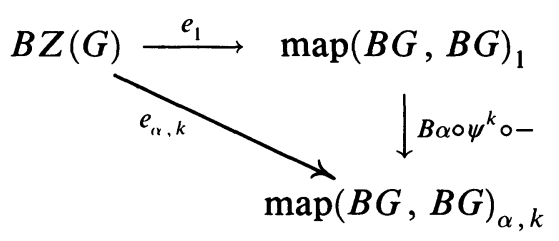

for some map $\psi^{k}$ of type $k$. Our description of the profinite homotopy type of $\operatorname{map}(B G, B G)$ is completed by the following theorem.

Theorem 2. For any connected simple $G$, the maps $e_{0}$, and $e_{\alpha, k}$ for each $\alpha$ and $k$, induce isomorphisms of homology with any finite coefficients.

We now outline the proof of Theorems 1 and 2. With the help of Sullivan's arithmetic square [11] for $B G$, the study of $\operatorname{map}(B G, B G)$ can be reduced to that of the mapping spaces $\operatorname{map}(B G, B G \hat{p})$ for all primes $p$ : where $(-) \hat{p}$ denotes the $p$-adic completion.

The key step is to approximate $B G$ at each prime $p$, as a homotopy colimit of $B P$ 's for certain $p$-toral subgroups $P \subseteq G$. This can in fact be done for any compact Lie group $G$, extending results in [6]. The indexing category for these limits is the full subcategory $\mathscr{R}_{p}(G)$ of the category of $G$-spaces, whose objects are the orbits $G / P$ for which

(i) $P \subseteq G$ is $p$-toral and 
(ii) $N(P) / P$ is finite and contains no nontrivial normal $p$-subgroups.

This is a category with finitely many isomorphism classes of objects and finite morphism sets. Let $\mathscr{I}: \mathscr{R}_{p}(G) \rightarrow G$-spaces be the inclusion functor, and let $E G \times{ }_{G} \mathscr{I}$ be its composite with the Borel construction. Note that $\left(E G \times_{G} \mathscr{I}\right)(G / H)=E G \times{ }_{G} G / H$ is homotopy equivalent to $B H$.

Theorem 3. For any compact Lie group $G$ and any prime $p$, $\underset{\text { hocolim}_{(\mathscr{R}}(G)}{\longrightarrow}(\mathscr{I})$ is $\mathbf{Z} /$ p-acyclic, and hence

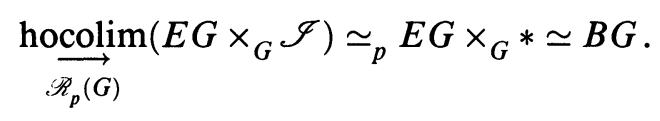

The main step when proving Theorem 3 is to construct a finite dimensional $\mathbf{Z} / p$-acyclic $G$-complex $X$, all of whose orbit types are in $\mathscr{R}_{p}(G)$. This is done using the approach in [9] and [10]. Since the fixed point set (hocolim $\left._{\mathscr{R}_{p}(G)}(\mathscr{I})\right)^{P}$ is contractible for any $G / P$ in $\mathscr{R}_{p}(G)$, there is a $G$-equivariant $\mathbf{Z} / p$-homology

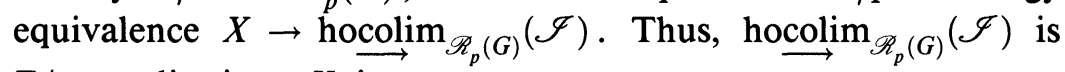
$\mathrm{Z} / p$-acyclic since $X$ is.

Recall that the main problem is to show that (2) implies (1) in Theorem 1; i.e., that any two maps $f, f^{\prime}: B G \rightarrow B G$ which are homotopic on $B T$ are homotopic on $B G$. For that, it suffices to show that they are homotopic inside $B G \hat{p}$ for each prime $p$. Using the theorems of Hubbuck [3,4] and Notbohm [7], we first show that if $f$ and $f^{\prime}$ are homotopic on $B T$, then they are homotopic on $B P$ for each prime $p$ and each $p$-toral subgroup $P \subseteq G$.

At this point, higher derived functors of inverse limits over $\mathscr{R}_{p}(G)$ begin to play a role. By a result of Wojtkowiak [14], if

$$
\lim _{\mathscr{R}_{p}(G)}^{i}\left(\pi_{j}\left(\operatorname{map}\left(E G \times_{G} \mathcal{I}, B G \hat{p}\right)_{f}\right)=0\right.
$$

for all $i, j>0$, then the inverse limit

$$
\varliminf_{\mathscr{R}_{p}(G)}\left(\operatorname{map}\left(E G \times_{G} \mathscr{I}, B G \hat{p}\right)_{f}\right)
$$

is also connected, and hence $f^{\prime}$ is homotopic to $f$. Also, in this case,

$$
\pi_{*}\left(\operatorname{map}(B G, B G \hat{p})_{f}\right) \cong \lim _{G / P \in \mathscr{R}_{p}(G)}\left(\pi_{*}\left(\operatorname{map}\left(E G \times_{G} \mathscr{I}, B G \hat{p}\right)_{f}\right)\right) .
$$


Note that

$$
\begin{aligned}
{\stackrel{\lim }{\mathscr{R}_{p}(G)}}^{i}\left(\pi_{j}\left(\operatorname{map}\left(E G \times_{G} \mathscr{J}, B G \hat{p}\right)_{f}\right)\right) \\
\quad=\underbrace{\lim ^{i}\left(\pi_{j}\left(\operatorname{map}(B P, B G \hat{p})_{f \mid B P}\right)\right)}_{G / P \in \mathscr{R}_{p}(G)}
\end{aligned}
$$

and similarly for the other limits.

The remaining step is now to show that these higher limits all vanish. The inverse systems are made explicit by the isomorphism

$$
\pi_{*}\left(\operatorname{map}(B P, B G \hat{p})_{f}\right) \cong \pi_{*}\left(B C_{G}(\rho) \hat{p}\right)
$$

of [2] and [8]: for $\rho: P \rightarrow G$ such that $B \rho \simeq f \mid B P$. In particular, if $(\alpha, k)$ is the type of $f$ (and $\left.f^{\prime}\right)$, we get that

$$
\pi_{*}\left(\operatorname{map}(B P, B G \hat{p})_{f \mid B P}\right) \simeq \begin{cases}\pi_{*}\left(B C_{G}(P)\right) \hat{p} & \text { if } k>0, \\ \pi_{*}(B G) \hat{p} & \text { if } k=0 .\end{cases}
$$

For components of type $(\alpha, k)$ with $k \neq 0$, Theorem 1 and 2 now follow from the following lemma:

Lemma 4. If $G$ is simple and $p$ is any prime, then

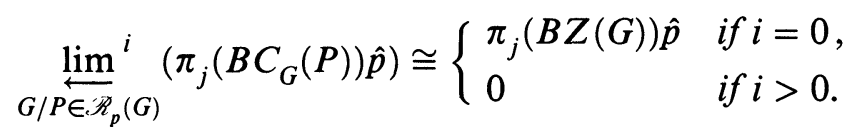

The proof of Lemma 4 uses a reinterpretation of derived functors over $\mathscr{R}_{p}(G)$. For any (contravariant) functor $F: \mathscr{R}_{p}(G) \rightarrow$ Groups, $\lim _{\mathscr{R}_{p}(G)}^{*}(F) \cong H_{G}^{*}\left(\operatorname{\text {hocolim}}_{\mathscr{R}_{p}(G)}(\mathscr{I}) ; F\right)$. Here, $H_{G}^{*}(-; F)$ denotes equivariant cellular cohomology (see [13]). This last group is closely related to $H_{G}^{*}(X ; F)$, where $X$ is the finite dimensional acyclic $G$ complex described after Theorem 3. The lemma is then proven by computing these cohomology groups for the given functors on $\mathscr{R}_{p}(G)$, using among other things the proof of the Conner conjecture in [10].

For $f$ of type 0 , Theorems 1 and 2 are special cases of the following theorem.

Theorem 5. Let $G$ and $\Gamma$ be any two compact Lie groups, and let $T$ be a maximal torus in $G$.

(i) $A$ map $f: B G \rightarrow B \Gamma$ is null homotopic if and only if $f \mid B N(T)$ is null homotopic.

(ii) If $G$ is connected, then $f$ is null homotopic if and only if $f \mid B T \simeq *$; or equivalently if and only if $\widetilde{H}^{*}(f ; \mathbf{Q})=0$.

(iii) $e_{0}: B \Gamma \rightarrow \operatorname{map}(B G, B \Gamma)_{0}$ induces an isomorphism of cohomology with arbitrary finite coefficients. 
Theorem 5 follows from the vanishing-for any $G$ and $p$-of higher limits of constant $p$-local functors over $\mathscr{R}_{p}(G)$. It is closely related to a theorem of Zabrodsky [15].

\section{REFERENCES}

1. W. Dwyer and G. Mislin, On the homotopy type of the components of $\operatorname{map}\left(B S^{3}, B S^{3}\right)$, Lecture Notes in Math., vol. 1298, Springer-Verlag, Berlin and New York, 1987, pp. 82-89.

2. W. Dwyer and A. Zabrodsky, Maps between classifying spaces, Lecture Notes in Math., vol. 1298, Springer-Verlag, Berlin and New York, 1987, pp. 106119.

3. J. Hubbuck, Homotopy homomorphisms of Lie groups, New Developments in Topology, Cambridge Univ. Press, 1974, pp. 33-41.

4. __ Mapping degrees for classifying spaces. I, Quart. J. Math. Oxford Ser. (2) 25 (1974), 113-133.

5. K. Ishiguro, Unstable Adams operations on classifying spaces, Math. Proc. Cambridge Philos. Soc. 102 (1987), 71-75.

6. S. Jackowski and J. McClure, Homotopy approximations for classifying spaces of compact Lie groups, Lecture Notes in Math., vol. 1370, Springer-Verlag, Berlin and New York, 1989, pp. 221-234.

7. G. Mislin, The homotopy classification of self-maps of infinite quaternionic projective space, Quart. J. Math. Oxford 38 (1987), 245-257.

8. D. Notbohm, Abbildungen zwischen klassifizierenden Räumen, dissertation, Göttingen, 1988.

9. R. Oliver, Smooth compact Lie group actions on disks, Math. Z. 149 (1976), 79-96. 10. $\frac{}{644 .}$, A proof of the Conner conjecture, Ann. of Math. (2) 103 (1976), 637-

11. D. Sullivan, Geometric topology, Part I: Localization, periodicity and Galois symmetry, Mimeographed notes, M.I.T., 1970.

12. C. W. Wilkerson, Self-maps of classifying spaces, Localization in group theory and homotopy theory, Lecture Notes in Math., vol. 418, Springer-Verlag, 1974, pp. 150-157.

13. S. Willson, Equivariant homology theories on G-complexes, Trans. Amer. Math. Soc. 212 (1975), 155-171.

14. Z. Wojtkowiak, On maps from holim $F$ to $Z$, Lecture Notes in Math., vol. 1298, Springer-Verlag, Berlin and New York, 1987, pp. 227-236.

15. A. Zabrodsky, Maps between classifying spaces, Algebraic topology and algebraic $K$-theory, Ann. of Math. Studies 113, Princeton Univ. Press, 1987, 228-246.

16. G. Carlsson, Equivariant stable homotopy and Sullivan's conjecture (to appear). 
17. J. Lannes, Sur la cohomology modulo $p$ des p-groupes abéliens élémentaires, Homotopy Theory, Proc. Durham Sympos. 1985, Cambridge Univ. Press, 1987, pp. 97-116.

18. H. Miller (to appear).

Institute of Mathematics, University of Warsaw, Pl00901 Warsaw, POLAND

Department of Mathematics, University of Kentucky, Lexington, KENTUCKY 40506-0027

Department of Mathematics, Aarhus University, Aarhus, Denmark 